\title{
Inhibition of mRNA Maturation by Compounds Which Have a Flavonoid Skeleton
}

\author{
Masashi Kurata $^{1,2}$, Mari Morimoto ${ }^{1}$, Yuko Kawamura ${ }^{3}$, Intisar Fouad Ali Mursi ${ }^{1}$, Keiko Momma ${ }^{4}$, \\ Masakazu Takahashi ${ }^{5}$, Yusaku Miyamae ${ }^{1}$, Taiho Kambe ${ }^{1}$, Masaya Nagao ${ }^{1}$, Hiroshi Narita ${ }^{3}$, \\ Yasuyuki Shibuya ${ }^{2}$, Seiji Masuda ${ }^{1, *}$ \\ ${ }^{1}$ Division of Integrated Life Sciences, Graduate School of Biostudies, Kyoto University, Kyoto, Japan \\ ${ }^{2}$ Department of Oral and Maxillofacial Surgery, Graduate School of Medical Sciences, Nagoya City University, Nagoya, Japan \\ ${ }^{3}$ Department of Food and Nutrition, Kyoto Women's University, Kyoto, Japan \\ ${ }^{4}$ Department of Living and Welfare, Kyoto Women's University, Kyoto, Japan \\ ${ }^{5}$ Department of Bioscience, Fukui Prefectural University, Fukui, Japan
}

\section{Email address:}

masuda@kais.kyoto-u.ac.jp (S. Masuda)

${ }^{*}$ Corresponding author

\section{To cite this article:}

Masashi Kurata, Mari Morimoto, Yuko Kawamura, Intisar Fouad Ali Mursi, Keiko Momma, Masakazu Takahashi, Yusaku Miyamae, Taiho Kambe, Masaya Nagao, Hiroshi Narita, Yasuyuki Shibuya, Seiji Masuda. Inhibition of mRNA Maturation by Compounds Which Have a Flavonoid Skeleton. Biochemistry and Molecular Biology. Vol. 2, No. 4, 2017, pp. 46-53. doi: 10.11648/j.bmb.20170204.13

Received: June 19, 2017; Accepted: June 29, 2017; Published: July 31, 2017

\begin{abstract}
Post-transcriptional modifications of nascent mRNA include 5' capping, splicing and 3' end polyadenylation, resulting in the emergence of mature mRNA. Recent findings indicate that mRNA splicing inhibitors can be potential anti-cancer candidates. Soy-isoflavone fractions displayed an inhibitory effect of mRNA processing among a number of dietary components. Two major components of the isoflavone fraction, daidzin and genistin did not have an inhibitory activity against mRNA maturation. The aglycone form of them also failed to inhibit mRNA maturation. Instead, compounds with flavone skeleton inhibited the mRNA maturation in the nucleus. Considering that the structural difference between flavone and isoflavone compounds is that B-ring is attached either on the 2' or 3' position of C-ring, respectively, anti-mRNA maturation activity may require a defined structural basis. These data indicate that compounds with flavone skeleton specifically alter the mRNA processing step.
\end{abstract}

Keywords: Flavone, Luciferase Reporter, RNA-FISH

\section{Introduction}

The maturation of mRNA is a multi-step process in the nucleus. This includes the critical steps of the 5' capping, splicing and 3' end-processing [1-5]. All of these processes are considered to be vital as well as strict quality control regulations that, eventually, aim to ensure the competency of the transcribed mRNA. Aberrantly transcribed mRNA will be retained in the nucleus to avoid the production of malfunctioning proteins [6-8]. During its life in the nucleus, mRNA is associated with a wide array of proteins which play vital roles influencing pre-mRNA processing by forming various complexes with it [1, 6-13]. Each step includes the rearrangement of the mRNA-protein complex. Inhibition of
mRNA processing functions as a potential strategy to investigate the dynamic transformations of mRNPs during different stages by obstructing their assembly at designated junctures. Messenger RNA splicing has received much attention recently as it was found to be central for the pathology of numerous diseases especially cancer. Therefore, mRNA splicing inhibition is thought to be an important therapeutic strategy [2, 14-21].

Several small compounds from natural resources which inhibit mRNA processing have been reported and it was proven that pre-mRNA splicing inhibition is the mechanistic foundation for their action [15, 16, 19, 22]. Spliceostatin A and pladienolide B inhibit mRNA splicing by binding to SF3b, a sub-complex of the $\mathrm{U} 2$ small nuclear ribonucleoprotein (U2 snRNA) in the spliceosome [23-25]. Gex1A also inhibits 
mRNA splicing via a similar mechanism to that of spliceostatin A and pladienolide B [26]. Isoginkgetin inhibits mRNA splicing by suppressing the rearrangement of spliceosomal complex A to complex B [27]. These compounds inhibit the spliceosomal formation and/or rearrangement. Other inhibitors with different mechanisms were also identified. NB-506, isodiospyrin and diospyrin inhibit the phosphorylation of SF2/ASF, a member of SR proteins that is required for mRNA splicing, through the inhibition of the kinase activity of topoisomerase I [28-30]. TG003 inhibits CLK kinase activity and also decreases the phosphorylation of SF2/ASF [31]. C77, an indole derivative, results in splicing inhibition that is mediated by SC35 and is not dependent on SF2/ASF [32]. These compounds affect the phosphorylation status of SR proteins. In addition, resveratrol derived from dietary resources such as grape skin and seeds, red wine, blueberries and rhubarb [33] alters the alternative splicing of certain types of mRNA [34], thereby suggesting there is an anti-mRNA processing inhibitory activity even in dietary origins. The diverse mechanisms by which the above mentioned compounds inhibit mRNA splicing highlight the complexity of this process. However, a limited number of inhibitors are still available up to date.

Previously, a monitoring system which is suitable for the screening of a wide range of chemical compounds that regulate mRNA processing has been established [35]. The first assay evaluates the activity of intron-containing Renilla luciferase reporter. The second assay measures the subcellular localization of mRNA (i.e. retention of pre-mRNA in the nucleus) by RNA- fluorescence in situ hybridization (FISH). Using this monitoring system, a number of samples from dietary origins were examined. The inhibitory activity of mRNA processing manifested by an isoflavone fraction that has been extracted from soybean was previously shown [36].

In the present study, compounds with flavone structure which were examined, exhibited anti-mRNA maturation activity. In addition, several compounds containing flavone skeletons also inhibited the mRNA processing The effect of the chemical structure on the activity of these compounds will be discussed.

\section{Materials and Methods}

\subsection{Cell Culture}

HeLa cells expressing Renilla luciferase (RLM1 cells) and U2OS (bone osteocarcinoma cell line) cells were maintained in Dulbecco's Modified Eagle's Medium (DMEM) (Wako, Tokyo, Japan) supplemented with $10 \%$ heat-inactivated fetal bovine serum at $37^{\circ} \mathrm{C}$.

\subsection{Measurement of Renilla Luciferase Activity}

In the first assay, $2 \times 10^{4}$ RLM1 cells previously established were inoculated in a 24-well plate and cultured for $24 \mathrm{~h} \mathrm{[35,}$ 37]. Sample was added in the culture media for $24 \mathrm{~h}$. The cells were washed with phosphate-buffered saline (PBS) and lysed by passive lysis buffer (Promega, Madison, WI) for $30 \mathrm{~min}$. The clear lysate obtained after spinning was used to measure the Renilla luciferase activity and the total protein content (Nacalai, Kyoto, Japan) according to the manufacturer's instructions. The Renilla luciferase activity was normalized with total protein.

\subsection{RNA Fluorescence in Situ Hybridization}

U2OS cells $\left(5 \times 10^{4}\right.$ cells $\left./ \mathrm{mL}\right)$ on coverslips in 12 well plate were cultured for $24 \mathrm{~h}$ after inoculation, fixed in $10 \%$ formaldehyde in PBS for $20 \mathrm{~min}$, and permeabilized in $0.1 \%$ Triton X-100 in PBS for $10 \mathrm{~min}$. Cells were washed three times with PBS for $10 \mathrm{~min}$ to remove the detergent and once with $2 \mathrm{x}$ standard sodium citrate (SSC) for $5 \mathrm{~min}$ to exchange the buffer content, prehybridized with ULTRAhyb-Oligo Hybridization Buffer (Ambion, Austin, TX) for $1 \mathrm{~h}$ at $42^{\circ} \mathrm{C}$ in a humidified chamber, and then incubated overnight with 10 pmol Cy3-labeled oligo-d $\mathrm{d}_{45}$ probe diluted with hybridization buffer. Cells were washed for $20 \mathrm{~min}$ at $42^{\circ} \mathrm{C}$ with $2 \mathrm{x} \mathrm{SSC}$, $0.5 \mathrm{x}$ SSC, and $0.1 \mathrm{x}$ SSC, respectively. The nuclei were visualized with 4', 6-diamidino-2-phenylindole (DAPI) [35, 38]. Quantification of the ratio of nuclear and cytoplasmic poly (A) ${ }^{+}$RNA signals was calculated with Image J software (https://imagej.nih.gov/ij/) according to the instructions.

\subsection{Cell Proliferation Assay}

U2OS cells were used to estimate the cytotoxic activity of each compound. The test samples were added to each cell cultured wells and incubated for $20 \mathrm{~h}$. Then, cell proliferation was assayed colorimetrically by using MTT (Sigma-Aldrich Japan, Tokyo, Japan). The cells were inoculated at $8 \times 10^{3}$ cells $/ \mathrm{mL}$ in a $96-$ well plate and cultured for $20 \mathrm{~h}$. After $5 \mu \mathrm{L}$ of MTT regent $(5 \mathrm{mg} / \mathrm{mL})$ was added to each well, the cells were incubated for $4 \mathrm{~h}$. The cell culture plate was centrifuged at 400 $\mathrm{x} g$ for $5 \mathrm{~min}$. The supernatant was removed. Then, the cells were solubilized with $10 \mathrm{mM} \mathrm{NH}_{4} \mathrm{Cl}$ containing $10 \%$ SDS ( $\mathrm{pH}$ 7.0). Cell proliferation was estimated by measuring the optical absorbance at $600 \mathrm{~nm}$.

\section{Results}

The inhibitory activity of mRNA from dietary origin was examined by the previously established monitoring system to assess the inhibition of mRNA processing including Renilla luciferase assay and RNA-FISH [35, 39]. The addition of soybean flavone fraction, Soya flavone HG (SHG) (Fuji Oil Co., Ltd.), increased the localization of mRNA in the nucleus, indicating that it contains an inhibitor of mRNA processing [36].

To obtain the active compound, SHG was separated by HPLC $\mathrm{C}_{18}$ column. The separated fraction was added to the cells according to the above mentioned method. Several fractions slightly increased the mRNA localization in the nucleus suggesting that the active compound will be more than one and the activity of each of them was much weaker compared to that of Gex1A, an inhibitor of mRNA splicing [26]. To find the active compound, known compounds in the 
isoflavone fraction and their derivatives were examined. Because a limited number of inhibitors are available to study the diverse mechanisms of mRNA processing, the identification of a new compound could lead to the discovery of a novel mRNA processing mechanism.
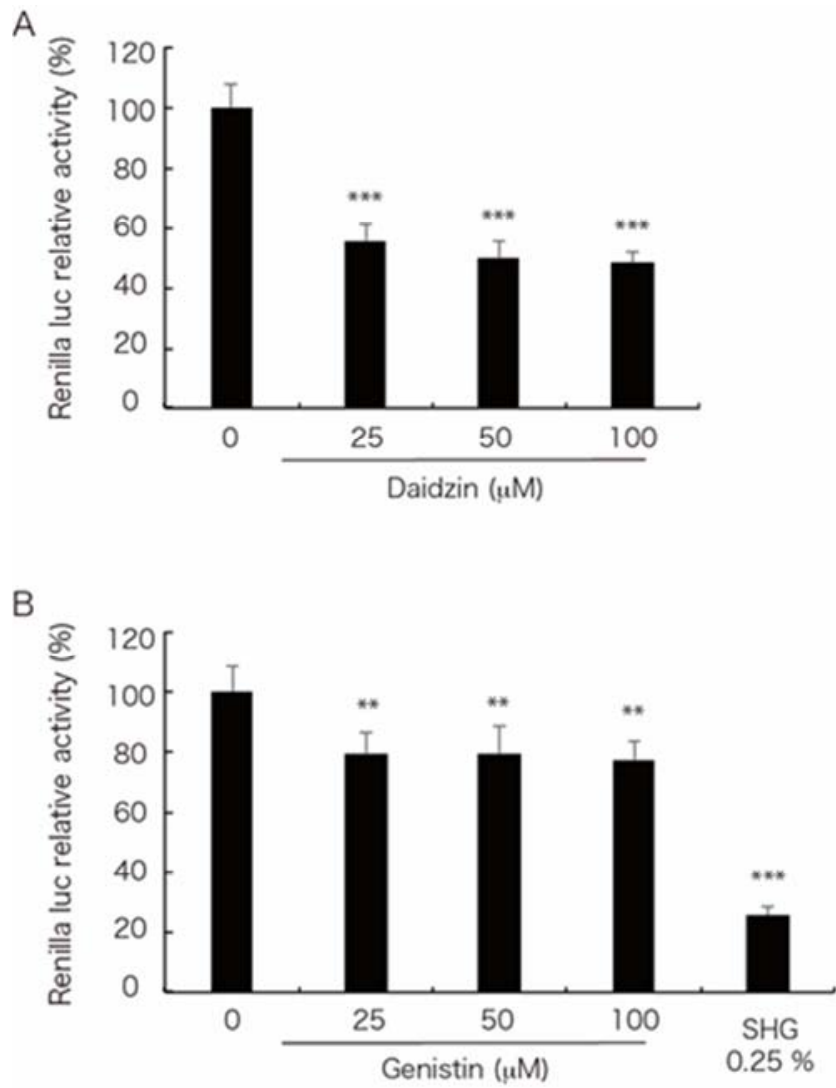

Figure 1. Daidzin and genistin decrease Renilla luciferase activity. A, Daidzin, B, Genistin. Renilla luciferase activity was measured and normalized by total protein using RLM1 cells stably expressing Renilla luciferase. Luciferase activity was shown as relative activity to control sample. SHG was used as a positive control. Each value is the mean $\pm S$. D. of quadruplicate experiments. Statistical analysis was performed using one-way ANOVA followed by Dunnett's test. P-values, **; $p<0.01$, ***; $p<0.001$.

The component composition of SHG is already shown previously [40]. Among them, the major constituents of the isoflavone fraction are daidzin, genistin and glycitin, and their derivatives including aglycone forms. To investigate whether the inhibition of mRNA processing was caused by these compounds, the Renilla luciferase activity was assessed. Daidzin clearly decreased the Renilla luciferase activity in a dose dependent manner (Figure 1). In contrast, genistin decreased the Renilla luciferase activity but its effect was little compared with the addition of $0.25 \%$ (weight/volume) SHG. To examine whether these compounds have an inhibitory activity of mRNA processing, RNA-FISH experiment was carried out to directly identify the localization of mRNA. Unexpectedly, daidzin did not alter the mRNA localization at all (Figure 2). Genistin also did not alter the mRNA localization. These results suggest that the decrease of Renilla luciferase activity by daidzin and genistin was not caused by the inhibition of mRNA maturation but by the inhibition of other processes.

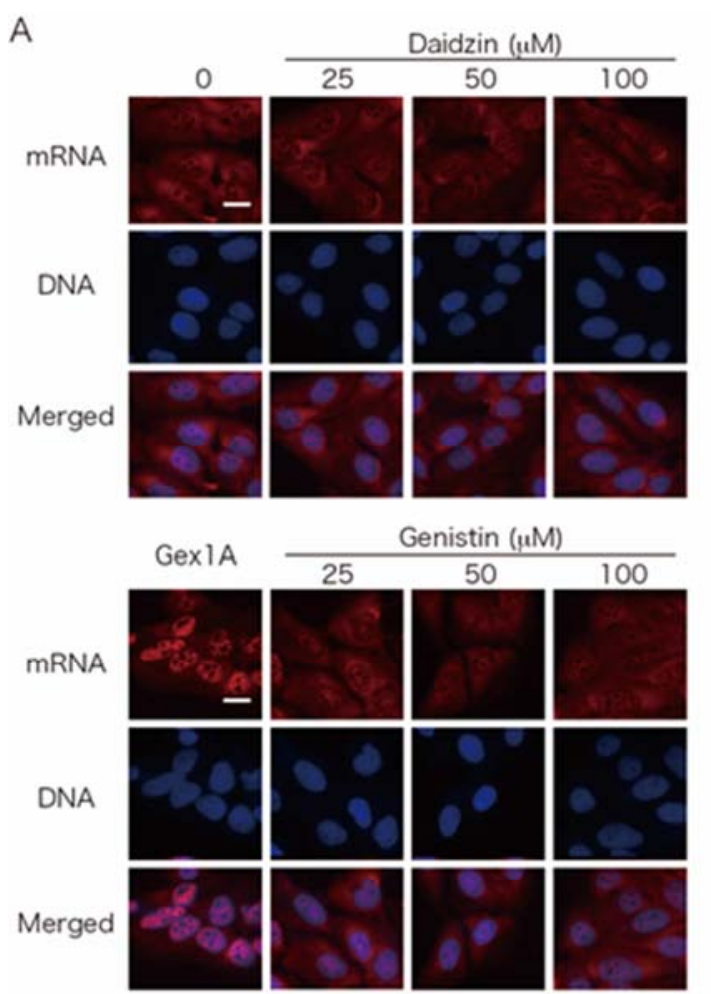

B

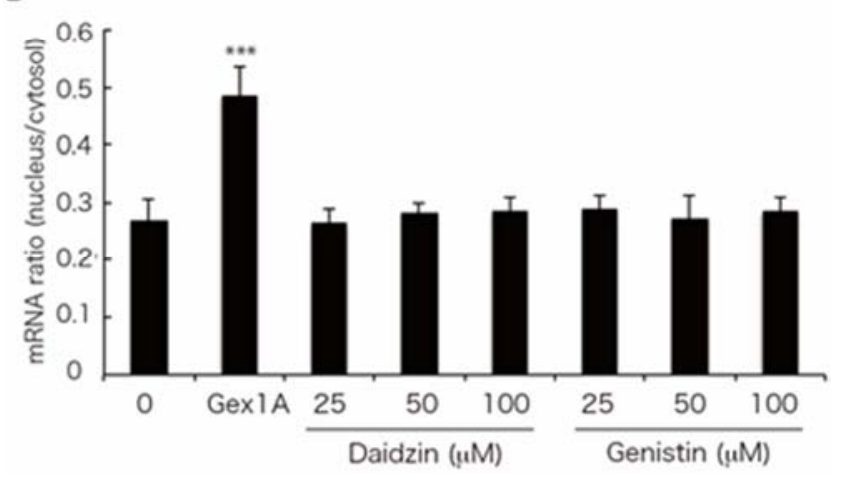

Figure 2. Daidzin and genistin did not inhibit the $m R N A$ maturation process. A, Subcellular distribution of $m R N A$. The bulk $m R N A$ was visualized by Cy3 labeled oligo $d T_{45}$ probe in U2OS cells. Cells were treated with indicated concentration of daidzin or genistin for $24 \mathrm{~h}$. Gex1 $\mathrm{A}(30 \mathrm{ng} / \mathrm{mL})$ was used as a positive control. The nuclei were stained with DAPI. B. The ratio of $m R N A$ localization. The signal intensity of the whole cell and that in the nucleus were quantified using ImageJ according to the manufacture's instruction. The cytoplasmic intensity was calculated by subtracting the nuclear intensity from the whole cellular intensity. Each value is the mean $\pm S . D . \quad(n=20)$. Statistical analysis was performed using one-way ANOVA followed by Dunnett's test. P-values, ***; $p<0$. 001. Scale bar, $10 \mu \mathrm{m}$.

In order to affect mRNA maturation, a candidate compound might be able to permeate through the cell membrane. As both daidzin and genistin are glycosylated, the sugar moieties potentiate their hydrophilic characters and therefore, impede both compounds' cellular penetration. To further explore this possibility, their aglycone form, daidzein and genistein were examined. But both daidzein and genistein failed to inhibit the Renilla luciferase activity 
(Figure 3). Instead, the addition of genistein slightly increased the activity. When the mRNA localization was observed by RNA-FISH, neither affected the mRNA localization even at high concentrations (Figure 4). These results indicate that the active compounds which inhibit the mRNA processing are found in insignificant amounts in SHG.


Figure 3. Effect of daidzein and genistein on Renilla luciferase activity. A, Daidzein, B, Genistein. Renilla luciferase activity was measured and normalized by total protein using RLM1 cells stably expressing Renilla luciferase. Luciferase activity was shown as relative activity to control sample. Gex $1 A(10 \mathrm{ng} / \mathrm{mL})$ was used as positive control. Each value is the mean $\pm S . D$. of triplicate experiments. Statistical analysis was performed using one-way ANOVA followed by Dunnett's test. P-values were not significant in each data.

Next, the possibility that minor component (s) have anti mRNA maturation activity was also examined. As daidzein and genistein gave negative results on mRNA maturation, various flavonoids were examined using the Renilla luciferase activity. Among the tested compounds, flavone (2-phenyl-1-benzopyran-4-one), which has one of the simplest structures, decreased the Renilla luciferase activity (Figure 5A). In addition, 7-hydroxyflavone and 7, 4'-dihydroxyflavone also inhibited the Renilla luciferase activity (Figure 5B-C). To examine whether these effects were caused by the inhibition of mRNA maturation, mRNA localization was observed by RNA-FISH experiments. Treatment with flavone slightly increased the mRNA localization in the nucleus in a dose-dependent manner (Figure 6A). 7-hydroxyflavone and 7, 4'-dihydroxyflavone also resulted in a gradual accumulation of mRNA in the nucleus (Figure 6B-C). The extent of mRNA accumulation is more obvious in 7-hydroxyflavone and 7, 4'-dihydroxyflavone than that in flavone (Figure 6D-F). These results indicate that some compounds with flavonoid skeleton have inhibitory effects on mRNA maturation process in the nucleus.

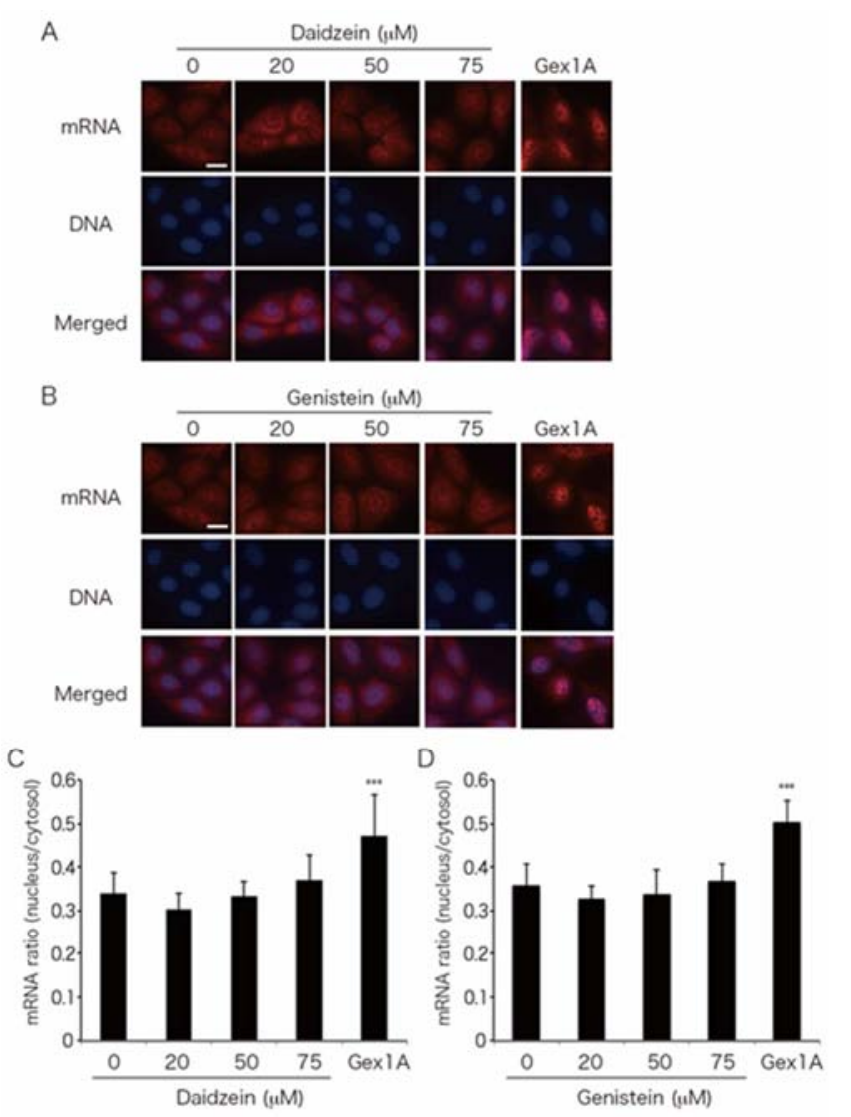

Figure 4. Daidzein and genistein did not inhibit the mRNA maturation process. $A$ and $B$, The bulk $m R N A$ was visualized by Cy3-labeled oligo $d T_{45}$ probe in U2OS cells. Cells were treated with indicated concentration of daidzein (A) and genistein (B) for 24 hours. GexlA $(30 \mathrm{ng} / \mathrm{mL})$ was used as positive control. The nuclei were stained with DAPI. C and D, Subcellular distribution of $m R N A$. The signal intensity of the whole cell and that in the nucleus were quantified using ImageJ according to the manufacture's instruction. The cytoplasmic intensity was calculated by subtracting the nuclear intensity from the whole cellular intensity. Each value is the mean $\pm S$. D. $(n=10)$. Statistical analysis was performed using one-way ANOVA followed by Dunnett's test. P-values, ***; $p<0$. 001. Scale bar, $10 \mu \mathrm{m}$.

To determine whether these compounds affected the cell proliferation, MTT assay was performed (Figure 7). The addition of flavone and 7-hydroxyflavone considerably inhibited the cell proliferation status. 7, 4'-dihydroxyflavone decreased the cell proliferation activity but its activity is less than flavone and 7-hydroxyflavone. These lines of evidence imply that these three compounds slow down the cell proliferation rate.

\section{Discussion}

The mRNA maturation process in the nucleus is a critical step for gene expression, cell proliferation and survival $[7,17$, 
18, 20, 21]. Inhibiting this process, therefore, significantly affects cell fate. Previously, several compounds with mRNA inhibitory activity were reported to be derived from the culture of microorganisms [24-26]. They interfered with the mRNA splicing through directly inhibiting the recruitment of key component (s) required for mRNA splicing or indirectly by modulating the phosphorylation status of essential SR proteins by inhibiting the kinase activity [28-32].
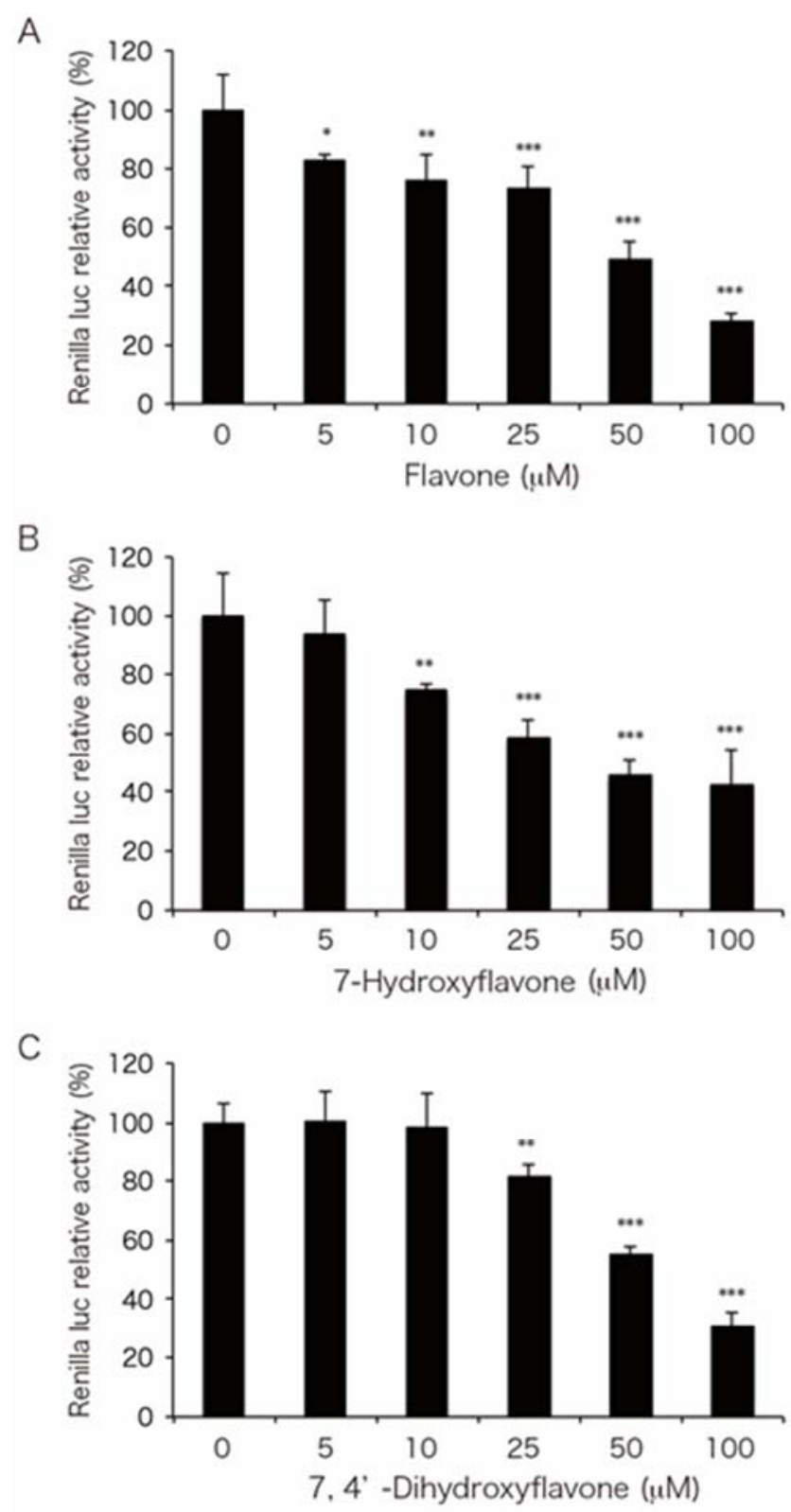

Figure 5. Flavone and its derivatives decreased Renilla luciferase activity. Renilla luciferase activity was measured and normalized by total protein using RLM1 cells stably expressing Renilla luciferase. Luciferase activity was shown as relative activity to control sample. A, Flavone, B, 7-hydroxyflavone, $C, 7,4$ '-dihydroxyflavone. Each value is the mean $\pm S$. D. of quadruplicate experiments. Statistical analysis was performed using one-way ANOVA followed by Dunnett's test. P-values, *; $p<0.05$, **; $p<0.01, * * * ; p<0$. 001 .

The presence of anti-mRNA maturation activity in SHG, a soybean isoflavone fraction, implies that chemical compounds which contain the isoflavone or related structures possess anti-mRNA maturation activity. To assess this hypothesis, several compounds with isoflavone and flavone skeleton were investigated. Two major components in the isoflavone fraction, daidzin and genistin, did not show prominent anti-mRNA maturation activity. The aglycone equivalents of daidzin and genistin, daidzein and genistein respectively, failed to show anti-mRNA maturation activity. In fact, genistein rather promoted the mRNA maturation process by enhancing Renilla luciferase activity as well as slightly decreasing the mRNA localization in the nucleus although their effect was not significant (Figure 3 and 4). These results imply that the active compound (s) is not a major component but will be found in insignificant amounts in the isoflavone fraction.


E
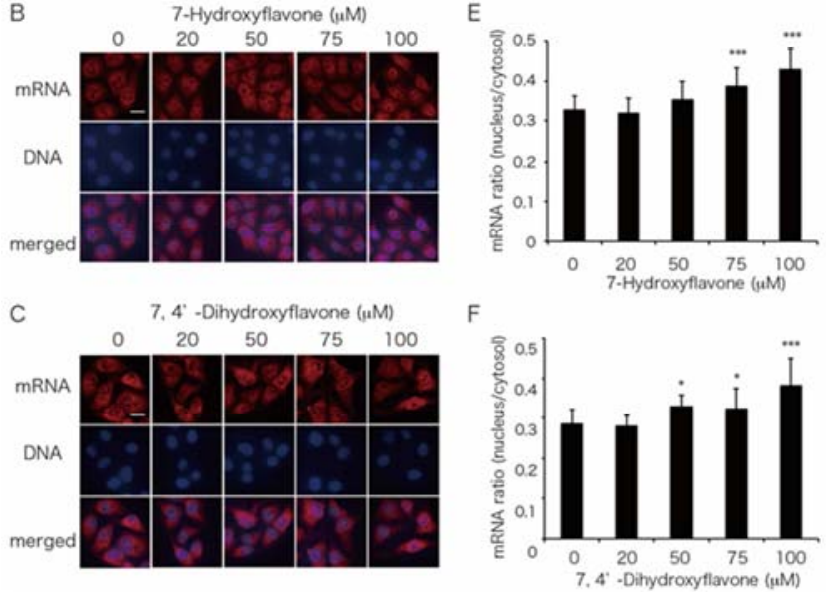

Figure 6. Flavone and its derivatives inhibited $m R N A$ maturation process. A-C, The bulk mRNA was visualized by Cy3-labeled oligo dT 45 probe in U2OS cells. Cells were treated with indicated concentration of each flavonoid for 24 h. The nuclei were stained with DAPI. A, flavone, B, 7-hydroxyflavone, C, 7, 4'-dihydroxyflavone. D-F, Subcellular distribution of $m R N A$. The signal intensity of the whole cell and that in the nucleus were quantified using ImageJ according to the manufacture's instruction. The cytoplasmic intensity was calculated by subtracting the nuclear intensity from the whole cellular intensity. D, flavone, E, 7-hydroxyflavone, F, 7, 4'-dihydroxyflavone. Each value is the mean $\pm S . D$. $(n=20)$. Statistical analysis was performed using one-way ANOVA followed by Dunnett's test. P-values, *; $p<0.05, * * ; p<0$. 01, ***; $p<0$. 001. Scale bar, $10 \mu \mathrm{m}$.

The effect of the compounds having a flavonoid skeleton on mRNA maturation was next examined. In order to screen the compound with flavone skeleton, three compounds to investigate their activity were chosen. Flavone has the simplest structure among the three candidates: a basic structure with a flavone skeleton. 7-Hydroxyflavone has one hydroxyl group in the A-ring. 7, 4'-dihydroxyflavone has two hydroxyl groups in the A- and C-rings. In this study, all of 
them with flavone skeleton showed weak but definite the anti-mRNA maturation activity. Instead, daidzein and genistein which both contain isoflavone skeleton failed to exhibit anti-mRNA maturation activity. These results indicate that the anti-mRNA maturation activity requires the certain structural basis and is not derived from the side effect such as general ant oxidative activity observed in flavonoids.

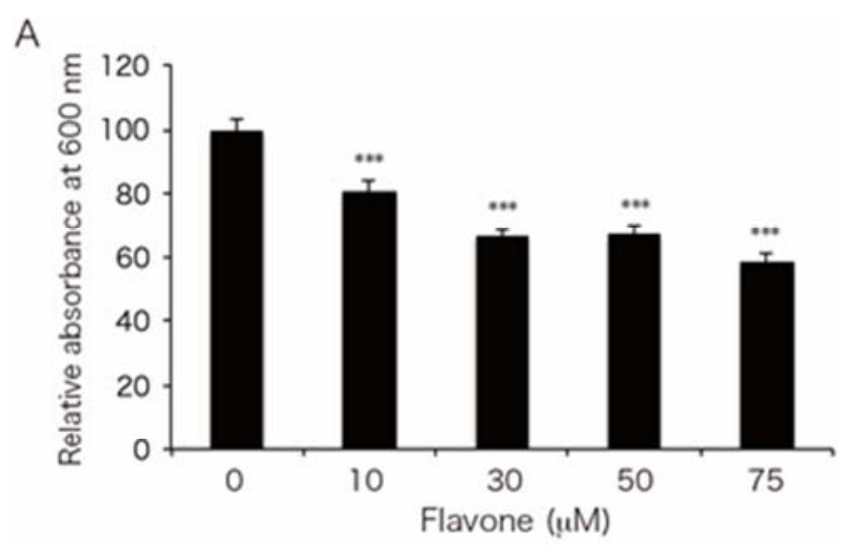

B

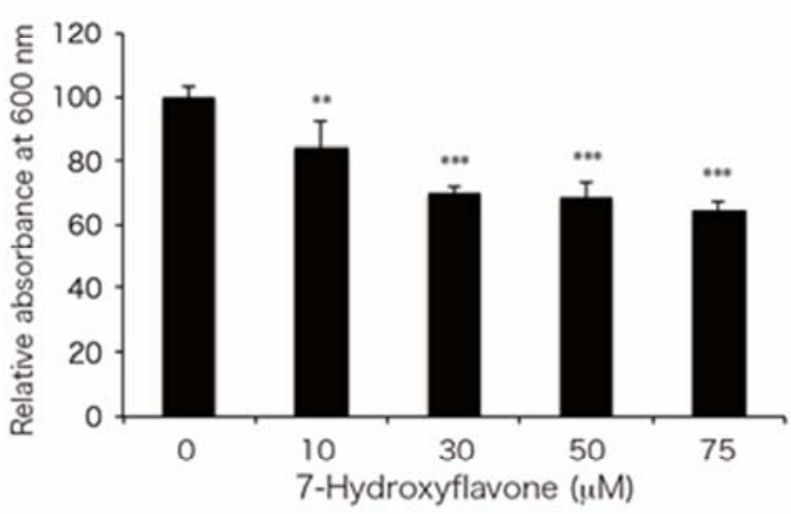

C

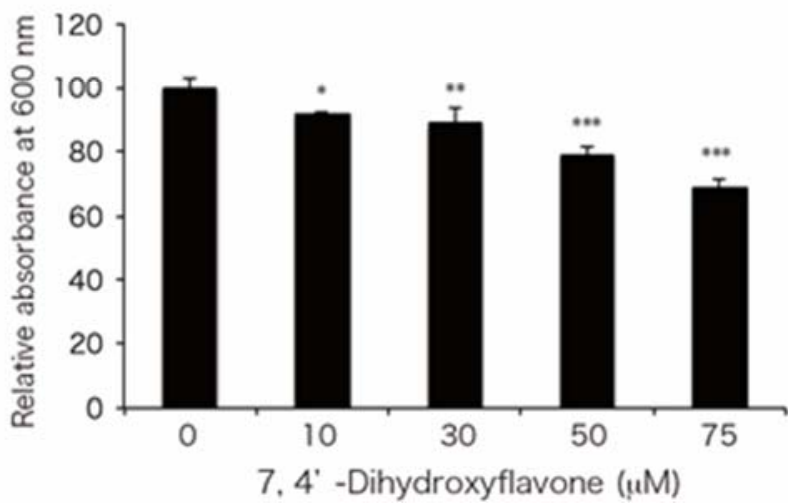

Figure 7. Flavone and its derivatives inhibit the cell proliferation. Flavonoids were added to U2OS cells for $24 \mathrm{~h}$, and then, MTT regent was added to cell culture media for $4 \mathrm{~h}$. The produced formazan was solubilized with the buffer containing $10 \mathrm{mM} \mathrm{NH}_{4} \mathrm{Cl}$ and $10 \% \mathrm{SDS}$. Flavone (A), 7-hydroxyflavone (B) and 7, 4'-dihydroxyflavone (C) were indicated, respectively. Each value is the mean $\pm S$. D. of triplicate experiments. Statistical analysis was performed using one-way ANOVA followed by Dunnett's test. P-values, *; $p<0.05$, **; $p<0.01,{ }^{* * *} ; p<0.001$.

The decrease of luciferase activity was more prominent with 7-hydroxyflavone, flavone and 7, 4'-hydroxyflavone, respectively. In the inhibition of mRNA maturation, 7-hydroxyflavone seems to be powerful, and 7, 4'-dihydroxyflavone comes next. Among the three compounds, flavone has the weakest activity in the inhibition of mRNA maturation. Nevertheless, flavone suppressed cell proliferation as much as 7-hydroxyflavone, suggesting it may have another activity affecting the cell growth other than the inhibition of mRNA maturation.

To affect the mRNA maturation step, compounds with flavonoid skeleton need to pass through both the cytoplasmic and nuclear membranes. Because small molecules with a molecular weight of $40 \mathrm{kDa}$ pass easily through the nuclear pore, small molecules, such as flavonoids, may easily enter into the nucleus [41-49]. But, a certain level or more hydrophobicity is necessary for a functional compound to pass through the cytoplasmic membrane. Moreover, polar functional groups, such as a hydroxyl group, attached to the specific site of the backbone structure of a compound often alter the affinity to its target protein. Such specific polar functional groups together with its backbone skeleton are essential for a certain pharmacokinetic and pharmacodynamic characteristics for each compound. Compared with the known mRNA splicing inhibitors, Gex1A, spliceostatin A and pladienolide $\mathrm{B}$, the three flavone compounds exhibited weak activity. Some molecular modification of the three flavone compounds based on the molecular comparison with the known mRNA splicing inhibitors like Gex1A may increase their inhibitory activity.

\section{Conclusion}

It was demonstrated that the recently established mRNA monitoring system is suitable to detect the activity of different compounds obtained from a number of resources including defined chemicals and natural products. The determination of the target processes for mRNA maturation (capping, splicing, 3' end-processing) and the target protein (s) by the three flavonoid compounds is also under investigation. Utilizing these compounds, deeper understanding of the detailed mechanism by which mRNA maturation inhibition occurs will be obtained. Clearly, it should be solved to validate the structure-activity relationship of the various derivatives in a flavone backbone and the effect they evoke in mRNA biogenesis.

\section{Acknowledgments}

The authors would like to express their gratitude to Kyowahakko company for providing Gex1A as a positive control reagent.

This work was supported in part by "grants-in-aid" from Central Miso Research Institute, Fuji Foundation for Protein Research, The Tojuro Iijima Foundation for Food Science and Technology, The Skylark Food Science Institute, The Foundation for Dietary Scientific Research and JSBBA Innovative Research Program Award to S. M. This work was 
supported in part by "grants-in-aid" from JSPS Kakenhi Grant Number $15 \mathrm{~K} 11299$ to Y. S.

\section{References}

[1] Chang, TH, Tung, L, Yeh, FL, et al. Functions of the $\mathrm{DExD} / \mathrm{H}-$ box proteins in nuclear pre-mRNA splicing, Biochim Biophys Acta. 2013; 1829:764-774.

[2] de Klerk, E, t Hoen, PA. Alternative mRNA transcription, processing, and translation: insights from RNA sequencing, Trends Genet. 2015; 31:128-139.

[3] Guo, J, Price, DH. RNA polymerase II transcription elongation control, Chem Rev. 2013; 113:8583-8603.

[4] Han, J, Xiong, J, Wang, D, et al. Pre-mRNA splicing: where and when in the nucleus, Trends Cell Biol. 2011;21:336-343.

[5] Wickramasinghe, VO, Laskey, RA. Control of mammalian gene expression by selective mRNA export, Nature reviews. Molecular cell biology. 2015; 16:431-442.

[6] Maniatis, T, Reed, R. An extensive network of coupling among gene expression machines, Nature. 2002;416:499-506.

[7] Naftelberg, S, Schor, IE, Ast, G, et al. Regulation of alternative splicing through coupling with transcription and chromatin structure, Annu Rev Biochem. 2015; 84:165-198.

[8] Saldi, T, Cortazar, MA, Sheridan, RM, et al. Coupling of RNA Polymerase II Transcription Elongation with Pre-mRNA Splicing, J Mol Biol. 2016; 428:2623-2635.

[9] Liu, YC, Cheng, SC. Functional roles of DExD/H-box RNA helicases in Pre-mRNA splicing, J Biomed Sci. 2015;22:54.

[10] Matera, AG, Wang, Z. A day in the life of the spliceosome, Nature reviews. Molecular cell biology. 2014; 15:108-121.

[11] Misra, A, Green, MR. From polyadenylation to splicing: Dual role for mRNA 3' end formation factors, RNA Biol. 2016; 13:259-264.

[12] Papasaikas, P, Valcarcel, J. The Spliceosome: The Ultimate RNA Chaperone and Sculptor, Trends Biochem Sci. 2016; 41:33-45.

[13] Sperling, J, Azubel, M, Sperling, R. Structure and function of the Pre-mRNA splicing machine, Structure. 2008; 16:1605-1615.

[14] Adamia, S, Pilarski, PM, Bar-Natan, M, et al. Alternative splicing in chronic myeloid leukemia (CML): a novel therapeutic target?, Curr Cancer Drug Targets. 2013; 13:735-748.

[15] Arslan, AD, He, X, Wang, M, et al. A high-throughput assay to identify small-molecule modulators of alternative pre-mRNA splicing, J Biomol Screen. 2013; 18:180-190.

[16] Bakkour, N, Lin, YL, Maire, S, et al. Small-molecule inhibition of HIV pre-mRNA splicing as a novel antiretroviral therapy to overcome drug resistance, PLoS Pathog. 2007; 3:1530-1539.

[17] Daguenet, E, Dujardin, G, Valcarcel, J. The pathogenicity of splicing defects: mechanistic insights into pre-mRNA processing inform novel therapeutic approaches, EMBO Rep. $2015 ; 16: 1640-1655$.
[18] Hahn, CN, Venugopal, P, Scott, HS, et al. Splice factor mutations and alternative splicing as drivers of hematopoietic malignancy, Immunol Rev. 2015; 263:257-278.

[19] Le, KQ, Prabhakar, BS, Hong, WJ, et al. Alternative splicing as a biomarker and potential target for drug discovery, Acta Pharmacol Sin. 2015; 36:1212-1218.

[20] Maguire, SL, Leonidou, A, Wai, P, et al. SF3B1 mutations constitute a novel therapeutic target in breast cancer, J Pathol. $2015 ; 235: 571-580$.

[21] Nlend Nlend, R, Meyer, K, Schumperli, D. Repair of pre-mRNA splicing: prospects for a therapy for spinal muscular atrophy, RNA Biol. 2010; 7:430-440.

[22] Salton, M, Misteli, T. Small Molecule Modulators of Pre-mRNA Splicing in Cancer Therapy, Trends Mol Med. 2016; 22:28-37.

[23] Folco, EG, Coil, KE, Reed, R. The anti-tumor drug E7107 reveals an essential role for SF3b in remodeling U2 snRNP to expose the branch point-binding region, Genes Dev. 2011; 25:440-444.

[24] Kaida, D, Motoyoshi, H, Tashiro, E, et al. Spliceostatin A targets SF3b and inhibits both splicing and nuclear retention of pre-mRNA, Nat Chem Biol. 2007; 3:576-583.

[25] Kotake, Y, Sagane, K, Owa, T, et al. Splicing factor SF3b as a target of the antitumor natural product pladienolide, Nat Chem Biol. 2007; 3:570-575.

[26] Hasegawa, M, Miura, T, Kuzuya, K, et al. Identification of SAP155 as the target of GEX1A (Herboxidiene), an antitumor natural product, ACS Chem Biol. 2011; 6:229-233.

[27] O'Brien, K, Matlin, AJ, Lowell, AM, et al. The biflavonoid isoginkgetin is a general inhibitor of Pre-mRNA splicing, J Biol Chem. 2008; 283:33147-33154.

[28] Pilch, B, Allemand, E, Facompre, M, et al. Specific inhibition of serine- and arginine-rich splicing factors phosphorylation, spliceosome assembly, and splicing by the antitumor drug NB-506, Cancer Res. 2001; 61:6876-6884.

[29] Tazi, J, Bakkour, N, Soret, J, et al. Selective inhibition of topoisomerase I and various steps of spliceosome assembly by diospyrin derivatives, Mol Pharmacol. 2005; 67:1186-1194.

[30] Ting, CY, Hsu, CT, Hsu, HT, et al. Isodiospyrin as a novel human DNA topoisomerase I inhibitor, Biochem Pharmacol. 2003; 66:1981-1991.

[31] Muraki, M, Ohkawara, B, Hosoya, T, et al. Manipulation of alternative splicing by a newly developed inhibitor of Clks, J Biol Chem. 2004; 279:24246-24254.

[32] Soret, J, Bakkour, N, Maire, S, et al. Selective modification of alternative splicing by indole derivatives that target serine-arginine-rich protein splicing factors, Proc Natl Acad Sci U S A. 2005; 102:8764-8769.

[33] Baur, JA, Sinclair, DA. Therapeutic potential of resveratrol: the in vivo evidence, Nat Rev Drug Discov. 2006; 5:493-506.

[34] Markus, MA, Marques, FZ, Morris, BJ. Resveratrol, by modulating RNA processing factor levels, can influence the alternative splicing of pre-mRNAs, PLoS One. 2011; 6:e28926.

[35] Fujita, K, Okamura, M, Nishimoto, S, et al. Establishment of a monitoring system to detect inhibition of mRNA processing, Biosci Biotechnol Biochem. 2012; 76:1248-1251. 
[36] Kurata, M, Murata, Y, Momma, K, et al. The isoflavone fraction from soybean presents the mRNA maturation inhibition activity., Biosci Biotechnol Biochem. 2017; 81:551-554

[37] Fujiwara, N, Yoshikawa, M, Yamazaki, T, et al. A screening method tuned for mRNA processing factors in human cells by evaluation of the luciferase reporter activity and the subcellular distribution of bulk poly (A)+ RNA, Biosci Biotechnol Biochem. 2010; 74:1512-1516.

[38] Masuda, S, Das, R, Cheng, H, et al. Recruitment of the human TREX complex to mRNA during splicing, Genes Dev. 2005; 19:1512-1517.

[39] Yamazaki, T, Fujiwara, N, Yukinaga, H, et al. The closely related RNA helicases, UAP56 and URH49, preferentially form distinct mRNA export machineries and coordinately regulate mitotic progression, Mol Biol Cell. 2010; 21:2953-2965.

[40] Kai, M, Yamauchi, A, Tominaga, K, et al. Soybean isoflavones eliminate nifedipine-induced flushing of tail skin in ovariectomized mice, J Pharmacol Sci. 2004; 95:476-478.

[41] Schwartz, TU. The Structure Inventory of the Nuclear Pore Complex, J Mol Biol. 2016; 428:1986-2000.
[42] Knockenhauer, KE, Schwartz, TU. The Nuclear Pore Complex as a Flexible and Dynamic Gate, Cell. 2016; 164:1162-1171.

[43] von Appen, A, Beck, M. Structure Determination of the Nuclear Pore Complex with Three-Dimensional Cryo electron Microscopy, J Mol Biol. 2016; 428:2001-2010.

[44] Hurt, E, Beck, M. Towards understanding nuclear pore complex architecture and dynamics in the age of integrative structural analysis, Curr Opin Cell Biol. 2015; 34:31-38.

[45] Kabachinski, G, Schwartz, TU. The nuclear pore complex--structure and function at a glance, J Cell Sci. 2015; 128:423-429.

[46] Ptak, C, Aitchison, JD, Wozniak, RW. The multifunctional nuclear pore complex: a platform for controlling gene expression, Curr Opin Cell Biol. 2014; 28:46-53.

[47] Grossman, E, Medalia, O, Zwerger, M. Functional architecture of the nuclear pore complex, Annu Rev Biophys. 2012; 41:557-584.

[48] Raices, M, D'Angelo, MA. Nuclear pore complex composition: a new regulator of tissue-specific and developmental functions, Nature reviews. Molecular cell biology. 2012; 13:687-699.

[49] Hoelz, A, Debler, EW, Blobel, G. The structure of the nuclear pore complex, Annu Rev Biochem. 2011; 80:613-643. 